\title{
Intake of fermented and non-fermented dairy products and risk of incident CHD: the Kuopio Ischaemic Heart Disease Risk Factor Study
}

\author{
Timo T. Koskinen, Heli E. K. Virtanen, Sari Voutilainen, Tomi-Pekka Tuomainen, Jaakko Mursu and \\ Jyrki K. Virtanen* \\ Institute of Public Health and Clinical Nutrition, University of Eastern Finland, P.O. Box 1627, 70211 Kuopio, Finland
}

(Submitted 27 February 2018 - Final revision received 29 August 2018 - Accepted 3 September 2018 - First published online 29 October 2018)

\begin{abstract}
Recent dairy product studies have suggested that fermented rather than non-fermented dairy products might provide benefits on cardiovascular health, but the evidence is inconclusive. Therefore, we investigated whether fermented and non-fermented dairy products have distinct associations with the risk of incident CHD in a population with high dairy product intake. The present study included a total of 1981 men, aged 42-60 years, from the Kuopio Ischaemic Heart Disease Risk Factor Study, with no CHD at baseline. Dietary intakes were assessed with instructed 4-d food records. We used Cox's proportional hazards regression model to estimate the associations with the risk of CHD. Fatal and non-fatal CHD events were ascertained from national registries. During a mean follow-up of $20 \cdot 1$ years, 472 CHD events were recorded. Median intakes were $105 \mathrm{~g} / \mathrm{d}$ for fermented ( $87 \%$ low-fat products) and $466 \mathrm{~g} / \mathrm{d}$ for non-fermented dairy products ( $60 \%$ low-fat products). After adjusting for potential confounders, those in the highest $(v$. lowest $)$ intake quartile of fermented dairy products had $27 \%(95 \% \mathrm{CI} 5,44 ; P$-trend $=0 \cdot 02)$ lower risk of CHD. In contrast, those in the highest intake quartile of non-fermented dairy products had $52 \%(95 \% \mathrm{CI} 13,104 ; P$-trend $=0.003)$ higher risk of CHD. When analysed based on fat content, low-fat $(<3.5 \%$ fat) fermented dairy product intake was associated with lower risk (hazard ratio in the highest quartile $=0.74 ; 95 \%$ CI $0.57,0.97 ; P$-trend $=0.03$ ), but high-fat fermented dairy and low-fat or high-fat non-fermented dairy products had no association. These results suggest that fermented and non-fermented dairy products can have opposite associations with the risk of CHD.
\end{abstract}

Key words: Dairy products: Fermented dairy products: CHD: Prospective cohort studies: Finnish men

CVD are the leading cause of death globally, CHD being the most common subtype ${ }^{(1)}$. According to the classic diet-heart hypothesis, SFA intake raises blood LDL-cholesterol, which increases the risk of CHD. Thus, the food industry has reduced the fat content especially in dairy foods, which are a major source of SFA with about two thirds of the dairy fat being SFA. In Finland, a country with a traditionally high dairy product intake, this change began concurrently with several lifestyle changes aimed to improve cardiovascular health, initiated by the North Karelia project in the $1970 \mathrm{~s}^{(2)}$. During the following decades, the consumption of low-fat and fat-free dairy products superseded full-fat dairy products ${ }^{(3)}$, and it has been suggested to have a beneficial effect on the blood cholesterol levels ${ }^{(4)}$. However, although there is convincing evidence that replacing SFA in the diet with unsaturated fatty acids has a favourable effect on the serum total and LDL-cholesterol ${ }^{(5)}$, the recent epidemiological studies suggest that the association of SFA intake with CHD risk may be modified by the food source, such as meats or dairy products. In these studies, dairy fat has not been independently associated with increased risk of $\mathrm{CHD}^{(6-8)}$. Similar lack of association with increased $\mathrm{CHD}$ risk has been observed in most epidemiological studies with intake of regular-fat or high-fat dairy products ${ }^{(9)}$, whereas some studies have observed that the associations may differ based on the type of dairy product consumed $^{(10,11)}$. For example, cheese and butter have had distinct associations with CHD, with cheese intake being associated with lower risk ${ }^{(10,11)}$.

In addition to epidemiological evidence, a few experimental studies have directly investigated the effects of substituting low-fat for high-fat dairy products. In a small ( $n$ 8) randomised crossover trial with 6-week intervention periods, skimmed milk intake improved total and LDL-cholesterol while whole milk intake showed no effect ${ }^{(12)}$, whereas in a more recent crossover trial ( $n$ 17), with 3-week intervention periods and no washout period, whole milk intake increased serum HDL-cholesterol concentration when compared with skimmed milk intake ${ }^{(13)}$. In addition, a 12-week, randomised, parallel design trial comparing different cheese varieties found that neither regular-fat nor reduced-fat cheese increased LDL-cholesterol $^{(14)}$. In a meta-analysis, cheese was found to have a blunted effect on total and LDL-cholesterol compared with butter ${ }^{(15)}$, despite similar fat content.

These findings suggest that dairy products should not be evaluated solely based on their fat content. Rather, the whole

Abbreviations: AR, absolute risk; HR, hazard ratio; KIHD, Kuopio Ischaemic Heart Disease Risk Factor Study.

* Corresponding author: J. K. Virtanen, fax +35817162 936, email jyrki.virtanen@uef.fi 
food matrix should be considered ${ }^{(16)}$. Based on experimental and prospective studies to date, it seems plausible that manufacturing processes, such as fermentation, could influence how different dairy products affect the development of $\mathrm{CVD}^{(10,17)}$. Fermentation process includes adding live bacteria to dairy foods, which can act as probiotics and result in multiple cardiometabolic benefits ${ }^{(18)}$. Some of these bacteria, used for example in cheese making, form vitamin $\mathrm{K}_{2}$, which has been associated with lower risk of CHD $^{(19,20)}$

Overall, the evidence concerning the impact of various dairy products on the CHD risk is inconclusive. Previous studies have mainly grouped dairy products based on their fat content or investigated commonly used single dairy items like milk, cheese or yogurt, while other studies have considered dairy foods as one group $^{(21)}$. Thus, to clarify the impact of dairy product consumption on CHD development, we aimed to investigate how different dairy product categories, based on fermentation status and fat content, are associated with the CHD risk. As the average dairy product intake in Finland is very high compared with the average intake worldwide ${ }^{(3,22)}$, we were able to investigate this question in the Kuopio Ischaemic Heart Disease Risk Factor Study (KIHD) cohort of middle-aged and older Finnish men.

\section{Methods}

\section{Study population}

The KIHD was designed to investigate risk factors for CVD, atherosclerosis, and related outcomes in a population-based, randomly selected sample of men from Eastern Finland ${ }^{(23)}$. The baseline examinations were conducted in 1984-1989. A total of 2682 men ( $83 \%$ of those eligible) were recruited in two cohorts. The first cohort consisted of 1166 men who were 54 years old, enrolled in 1984-1986, and the second cohort included 1516 men who were $42,48,54$ or 60 years old, enrolled in 1986-1989. Re-examination rounds were conducted 4 and 11 years after the baseline (online Supplementary Fig. S1). The baseline characteristics of the entire study population have been described previously ${ }^{(23)}$. Research Ethics Committee of the University of Kuopio has approved the KIHD study protocol. All subjects gave written informed consent for participation. The dietary compound of the KIHD has been registered at Clinicaltrials.gov (identifier: NCT 03221127).

We excluded subjects with history of CHD ( $n$ 677) at baseline, or with missing data on dietary intakes ( $n$ 24), leaving 1981 men for the analyses of incident CHD.

\section{Dietary assessment}

Dietary intakes were assessed at baseline, using 4-d food records including one weekend day. A nutritionist instructed participants on recording food consumption in household measurement units at baseline. To help in the estimation of portion sizes, participants received a picture book of common foods and dishes. For each food item, the participant could choose from 3 to 5 commonly used portion sizes or describe the portion size in relation to examples from the book. To further improve accuracy, a nutritionist checked the completed records together with the participant.

Food and nutrient intakes were estimated from the food records using the NUTRICA ${ }^{\circledR} 2.5$ software (Social Insurance Institution). The databank of the software is mainly based on Finnish values of nutrient composition of foods. The four main dairy food groups used in the calculations were low-fat and high-fat fermented and non-fermented dairy products (Table 1). Dairy products with $<3.5 \%$ fat were considered as low-fat. Colostrum was excluded from high-fat milk group because it does not go through the same manufacturing process as other milk. Total dairy product intake was calculated as the sum of fermented and non-fermented dairy products. Butter was excluded from these dairy product groups and analysed separately. Butter intake included butter from spreads containing butter, and butter from recipes.

\section{Other measurements}

Venous blood samples were collected between 08.00 and 10.00 hours at the baseline examinations. Subjects were instructed to fast and abstain from smoking for $12 \mathrm{~h}$ before, and to abstain from alcohol for $3 \mathrm{~d}$ before providing the samples. Detailed descriptions of the determination of serum lipids and lipoproteins, assessment of medical history and medications at baseline, family history of diseases, education and income, smoking, alcohol intake, blood pressure, serum fatty acids, and physical activity have been published elsewhere ${ }^{(24-26)}$. Serum C-reactive protein was measured with an immunometric assay (Immulite High Sensitivity CRP Assay; Diagnostic Products Corporation). Diabetes was defined as selfreported diabetes mellitus or fasting blood glucose over $6.9 \mathrm{mmol} / \mathrm{l}$. Hypertension was defined as systolic/diastolic blood pressure $\geq 140 / 90 \mathrm{mmHg}$ or the use of hypertension medication. Family history of CHD was defined as positive if a first-degree relative of the participant had a history of CHD.

\section{Ascertainment of follow-up events}

Data on fatal and non-fatal coronary events from the beginning of study till the end of 2012 were obtained by computer linkage to the national hospital discharge and death certificate registers. Diagnostic information was collected from hospitals and classified using identical diagnostic criteria. Each suspected coronary event (International Classification of Diseases (ICD)-9 codes 410-414 and ICD-10 codes I20-I25) was classified into (1) a definite acute myocardial infarction, (2) a probable acute myocardial infarction, (3) a typical acute chest pain episode of more than 20 min indicating CHD, (4) an ischaemic cardiac arrest with successful resuscitation or (5) no acute coronary event by a physician using the original patient records. If the subject had multiple non-fatal coronary events during the follow-up period, the first event was considered the end point.

\section{Statistical analysis}

The univariate relationships between fermented or non-fermented dairy product intake and baseline characteristics were assessed by means and linear regression for continuous variables or $\chi^{2}$-tests for categorical variables. We used Cox's proportional 
Table 1. Dairy foods included in low-fat and high-fat fermented and non-fermented dairy food groups with mean intakes $(\mathrm{g} / \mathrm{d})$

(Mean values, standard deviations and percentages)

\begin{tabular}{|c|c|c|c|}
\hline & Mean & SD & $\%$ \\
\hline Fermented dairy products & 187 & 218 & $26^{*}$ \\
\hline Low-fat fermented dairy products ( $<3.5 \%$ fat) & 162 & 218 & $87 \dagger$ \\
\hline Sour milk $(\leq 2.5 \%$ fat $)$ & 136 & 206 & 72 \\
\hline Low-fat cultured buttermilk ( $\leq 2.5 \%$ fat) & 11 & 38 & 6 \\
\hline Low-fat yogurt $(\leq 2.5 \%$ fat $)$ & 11 & 36 & 6 \\
\hline Fat-free yogurt & 0.3 & 5 & 0.2 \\
\hline Kefir & 2 & 25 & 1 \\
\hline Curd cheese/quark & 1 & 6 & 0.5 \\
\hline Cottage cheese & 0.4 & 4 & 0.2 \\
\hline High-fat fermented dairy products ( $\geq 3.5 \%$ fat) & 25 & 29 & 13 \\
\hline Cheeseł & 22 & 26 & 12 \\
\hline Crème fraîche and sour cream & 2 & 11 & 1 \\
\hline High-fat cultured buttermilk & 0.3 & 6 & 0.2 \\
\hline High-fat yogurt & 0.1 & 4 & 0.1 \\
\hline Non-fermented dairy products & 520 & 336 & $74^{*}$ \\
\hline Low-fat non-fermented dairy products ( $<3.5 \%$ fat) & 314 & 291 & $60 \dagger$ \\
\hline Low-fat milk§ ( $\leq 1.9 \%$ fat $)$ & 314 & 291 & 60 \\
\hline Fat-free milk & 46 & 146 & 9 \\
\hline Powdered milk and milk-based convenience food soups & 0.3 & 5 & 0.1 \\
\hline High-fat non-fermented dairy products ( $\geq 3.5 \%$ fat) & 205 & 300 & 39 \\
\hline High-fat milk\| & 180 & 296 & 35 \\
\hline Cream & 17 & 28 & 3 \\
\hline Ice cream & 5 & 13 & 1 \\
\hline Colostrum & 3 & 18 & 0.6 \\
\hline Bread cheese/Finnish squeaky cheese & 0.3 & 4 & $0 \cdot 1$ \\
\hline
\end{tabular}

* Proportion of total dairy product intake.

† Proportion of total fermented or non-fermented dairy product intake.

‡ Includes all fermented cheese products like Blue, Edam, Gouda and Swiss cheese.

$\S$ Includes semi-skimmed milk and skimmed milk.

II Includes farm milk, whole milk and cream milk.

hazards regression model to estimate hazard ratios (HR) and $95 \%$ CI of dairy product groups in quartiles. Because the fermented dairy product subgroups and the high-fat milk subgroup included substantial number of non-users, we assigned the nonusers into the reference group and divided the rest into three equal groups. The validity of the proportional hazards assumption was confirmed with Schoenfeld residuals.

The main analyses included three models. Model 1 was adjusted for age (year), examination year and energy intake (kJ/d). Model 2 included additional adjustment for family history of CHD (yes/no), smoking (cigarette packs/day $\times$ years of smoking), education (year), leisure-time physical activity $(\mathrm{kJ} / \mathrm{d})$, energy adjusted intake of PUFA, and intakes of fruits, berries and vegetables $(\mathrm{g} / \mathrm{d})$, fibre $(\mathrm{g} / \mathrm{d})$ and alcohol $(\mathrm{g} /$ week). Finally, model 3 was further adjusted for potential effect mediators: BMI $\left(\mathrm{kg} / \mathrm{m}^{2}\right)$, diabetes (yes/no) and hypertension (yes/no). All quantitative variables were entered in the models as continuous variables. We used the cohort mean to replace missing values in covariates $(<2.0 \%)$. Change in absolute risk (AR) was calculated by multiplying the AR in the reference group by the increase or decrease in the multivariable-adjusted HR in the comparison group. Tests of linear trend were conducted by assigning the median values for each category of exposure variable and treating those as a single continuous variable. All $P$-values were two-tailed ( $\alpha=0 \cdot 05)$.

When assessing the substitution of fermented for nonfermented dairy food, we included the two groups simultaneously in the model and used the difference of regression coefficients of the two groups of interest and their variance and covariance to calculate the HR and 95\% CI.

Data were analysed using SPSS 21.0 for Windows (IBM Corp.).

\section{Results}

Table 2 shows the baseline characteristics according to fermented and non-fermented dairy product intake. Compared with men in the lowest quartile of fermented dairy product intake, men in the highest quartile were more likely to live in a rural area and less likely to be current smokers. They also had higher intakes of energy, fibre, and fruits, berries and vegetables, and lower intakes of MUFA and PUFA, and consumed less alcohol. Compared with men in the lowest quartile of non-fermented dairy product intake, men in the highest quartile were more likely to live in a rural area and to be current smokers, and less likely to be physically active or married, and they had fewer years of education. In addition, they had higher intakes of energy, fibre and SFA, and lower intakes of MUFA and PUFA, and fruits, berries and vegetables. Baseline characteristics based on low-fat and high-fat fermented and non-fermented dairy product intakes are presented in online Supplementary Tables S1 and S2.

Table 1 shows the mean intakes for the different fermented and non-fermented dairy product groups. The mean intake of total dairy foods was 707 (SD 365) g/d, of which 26\% (187 (sD 218) g/d) came from fermented and $74 \%(520(\mathrm{sD} 336) \mathrm{g} / \mathrm{d})$ from nonfermented dairy products (Table 1 ). Low-fat products comprised 
Table 2. Baseline characteristics of 1981 participants in the Kuopio Ischaemic Heart Disease Risk Factor Study in 1984-1989 according to fermented and non-fermented dairy product intake (Mean values and standard deviations)

\begin{tabular}{|c|c|c|c|c|c|c|c|c|c|c|c|c|c|c|c|c|}
\hline \multirow{3}{*}{$\begin{array}{l}\text { Intake quartile (intake range, } \\
\mathrm{g} / \mathrm{d} \text { ) }\end{array}$} & \multicolumn{8}{|c|}{ Fermented dairy products } & \multicolumn{8}{|c|}{ Non-fermented dairy products } \\
\hline & \multicolumn{2}{|c|}{$1(0-26)$} & \multicolumn{2}{|c|}{$2(26-105)$} & \multicolumn{2}{|c|}{$3(106-279)$} & \multicolumn{2}{|c|}{$4(280-1643)$} & \multicolumn{2}{|c|}{$1(0-258)$} & \multicolumn{2}{|c|}{$2(258-465)$} & \multicolumn{2}{|c|}{$3(466-727)$} & \multicolumn{2}{|c|}{$4(728-2394)$} \\
\hline & Mean & SD & Mean & SD & Mean & SD & Mean & SD & Mean & SD & Mean & SD & Mean & SD & Mean & SD \\
\hline Subjects $(n)$ & \multicolumn{2}{|c|}{501} & \multicolumn{2}{|c|}{494} & \multicolumn{2}{|c|}{492} & \multicolumn{2}{|c|}{494} & \multicolumn{2}{|c|}{495} & \multicolumn{2}{|c|}{495} & \multicolumn{2}{|c|}{496} & \multicolumn{2}{|c|}{495} \\
\hline Age (years) & $52 \cdot 6$ & $5 \cdot 3$ & $51 \cdot 7$ & 5.8 & 52.5 & $5 \cdot 2$ & $53 \cdot 1^{*}$ & 4.8 & $52 \cdot 0$ & 5.6 & 52.5 & $5 \cdot 2$ & $52 \cdot 7$ & $5 \cdot 2$ & $52 \cdot 6$ & $5 \cdot 1$ \\
\hline $\mathrm{BMI}\left(\mathrm{kg} / \mathrm{m}^{2}\right)$ & $27 \cdot 0$ & 4.0 & $26 \cdot 4$ & 3.2 & $26 \cdot 6$ & 3.2 & $26 \cdot 9$ & 3.6 & $26 \cdot 8$ & 3.5 & $26 \cdot 7$ & 3.6 & $26 \cdot 8$ & 3.6 & $26 \cdot 6$ & 3.4 \\
\hline $\begin{array}{l}\text { Leisure-time physical activity } \\
(\mathrm{kJ} / \mathrm{d})\end{array}$ & 502 & 669 & 623 & 770 & 628 & 724 & 565 & 636 & 628 & 674 & 582 & 623 & 611 & 732 & $494^{\star}$ & 761 \\
\hline Income (euro) & 12766 & 8565 & 16288 & 10486 & 15086 & 10261 & $12596^{\star}$ & 7342 & 17329 & 11935 & 13774 & 8012 & 13141 & 7445 & $12443^{*}$ & 8682 \\
\hline Education (years) & 8.6 & 3.4 & 9.9 & 4.0 & 9.3 & 3.9 & $8 \cdot 1^{*}$ & $2 \cdot 9$ & 9.9 & 3.9 & 9.0 & $3 \cdot 7$ & 8.9 & 3.6 & $8 \cdot 1^{*}$ & $3 \cdot 1$ \\
\hline Married (\%) & \multicolumn{2}{|c|}{83} & \multicolumn{2}{|c|}{90} & \multicolumn{2}{|c|}{88} & 87 & 7 & 91 & & 87 & & 85 & & $84^{*}$ & \\
\hline Living in rural area (\%) & 3 & 1 & 2 & 0 & 23 & & $35^{\star}$ & & $1 \varepsilon$ & & 28 & & 24 & & $39^{*}$ & \\
\hline Diabetes (\%) & 5 & & 4 & t & 4 & & 6 & & 6 & & 5 & & 4 & & 4 & \\
\hline Family history of $\mathrm{CHD}(\%)$ & 46 & & 4 & 4 & 47 & & 47 & & 46 & & 47 & & 49 & & 42 & \\
\hline Current smoker (\%) & $3 \varepsilon$ & 8 & 2 & 9 & 23 & & 24 & $l^{*}$ & 20 & & 26 & & 31 & & $36^{\star}$ & \\
\hline Hypertension (\%) & 57 & 7 & 5 & 1 & 5 & & 58 & 8 & 57 & & 54 & & 60 & & 53 & \\
\hline $\begin{array}{l}\text { Systolic blood pressure } \\
(\mathrm{mmHg})\end{array}$ & 135 & 17 & 133 & 16 & 134 & 17 & 136 & 16 & 134 & 16 & 134 & 16 & 135 & 17 & 134 & 17 \\
\hline $\begin{array}{l}\text { Diastolic blood pressure } \\
(\mathrm{mmHg})\end{array}$ & 90 & 11 & 88 & 10 & 89 & 11 & 90 & 10 & 89 & 10 & 89 & 10 & 89 & 11 & 89 & 11 \\
\hline $\begin{array}{l}\text { Fasting blood glucose } \\
(\mathrm{mmol} / \mathrm{l})\end{array}$ & $4 \cdot 8$ & 1.0 & $4 \cdot 7$ & 0.9 & 4.7 & 1.0 & $4 \cdot 7$ & $1 \cdot 0$ & $4 \cdot 7$ & 0.9 & $4 \cdot 7$ & 1.0 & $4 \cdot 7$ & $1 \cdot 1$ & $4 \cdot 7$ & 0.9 \\
\hline $\begin{array}{l}\text { Serum LDL-cholesterol } \\
(\mathrm{mmol} / \mathrm{l})\end{array}$ & 4.07 & 1.01 & 3.89 & 0.93 & 4.00 & 1.03 & 4.05 & 0.98 & 3.92 & 0.99 & 4.05 & 1.00 & 3.99 & 1.01 & 4.05 & 0.95 \\
\hline $\begin{array}{l}\text { Serum HDL-cholesterol } \\
(\mathrm{mmol} / \mathrm{l})\end{array}$ & 1.30 & 0.30 & 1.30 & 0.29 & 1.32 & 0.29 & 1.30 & 0.30 & 1.30 & 0.31 & 1.30 & 0.30 & 1.30 & 0.29 & 1.32 & 0.28 \\
\hline Serum TAG $(\mathrm{mmol} / \mathrm{l})$ & 1.28 & 0.76 & $1 \cdot 19$ & 0.62 & 1.26 & 0.83 & 1.28 & 0.73 & 1.31 & 0.79 & 1.23 & 0.67 & 1.27 & 0.82 & 1.21 & 0.67 \\
\hline C-reactive protein (mg/l) & 2.5 & 4.5 & $2 \cdot 1$ & $2 \cdot 9$ & $2 \cdot 2$ & 4.9 & $2 \cdot 2$ & 4.2 & $2 \cdot 0$ & 3.4 & $2 \cdot 3$ & 5.5 & $2 \cdot 2$ & 3.9 & $2 \cdot 4$ & 3.7 \\
\hline $\begin{array}{l}\text { Serum long-chain } n-3 \text { PUFA } \\
(\%)\end{array}$ & $4 \cdot 6$ & 1.6 & $4 \cdot 7$ & 1.6 & $4 \cdot 8$ & $1 \cdot 7$ & $4 \cdot 7$ & 1.6 & $5 \cdot 0$ & $1 \cdot 7$ & $4 \cdot 7$ & $1 \cdot 7$ & $4 \cdot 6$ & 1.5 & $4 \cdot 4^{*}$ & 1.4 \\
\hline Alcohol intake (g/week) & 81 & 137 & 82 & 125 & 61 & 86 & $68^{*}$ & 103 & 82 & 120 & 72 & 116 & 66 & 92 & 72 & 128 \\
\hline Dietary intakes & & & & & & & & & & & & & & & & \\
\hline Energy (kJ/d) & 10121 & 2628 & 10305 & 2498 & 10020 & 2602 & $10887^{\star}$ & 2573 & 8966 & 2230 & 9962 & 2339 & 10343 & 2167 & $12058^{*}$ & 2619 \\
\hline Protein (E\%) & $15 \cdot 0$ & 2.4 & 15.5 & $2 \cdot 2$ & 15.9 & 2.7 & $16 \cdot 5^{\star}$ & 2.4 & $15 \cdot 9$ & $2 \cdot 8$ & $15 \cdot 7$ & 2.6 & $15 \cdot 7$ & 2.4 & $15 \cdot 6$ & $2 \cdot 1$ \\
\hline Carbohydrates (E\%) & $42 \cdot 3$ & 6.9 & 42.5 & 6.6 & $43 \cdot 1$ & $6 \cdot 1$ & $43 \cdot 6^{*}$ & $5 \cdot 7$ & $42 \cdot 7$ & $7 \cdot 1$ & 43.5 & $6 \cdot 3$ & $43 \cdot 2$ & $6 \cdot 1$ & $42 \cdot 2$ & 5.9 \\
\hline Fibre $(g / d)$ & $24 \cdot 3$ & 8.8 & $25 \cdot 0$ & 8.6 & 24.8 & $8 \cdot 6$ & $27 \cdot 6^{\star}$ & 8.5 & $23 \cdot 8$ & $8 \cdot 3$ & $25 \cdot 2$ & $8 \cdot 7$ & $25 \cdot 2$ & $8 \cdot 2$ & $27.5^{\star}$ & $9 \cdot 3$ \\
\hline Total fat (E\%) & 39.6 & 6.4 & 38.9 & 5.8 & 38.5 & 5.6 & $37.5^{\star}$ & $5 \cdot 2$ & 37.8 & $6 \cdot 0$ & $38 \cdot 1$ & 5.5 & 38.6 & 5.6 & $39.9^{\star}$ & 5.9 \\
\hline PUFA (E\%) & 4.6 & 1.5 & 4.7 & 1.4 & 4.6 & 1.4 & $4 \cdot 2^{*}$ & 1.2 & $5 \cdot 1$ & 1.5 & 4.7 & 1.4 & 4.4 & 1.3 & $3 \cdot 9^{*}$ & $1 \cdot 1$ \\
\hline MUFA (E\%) & $12 \cdot 1$ & $2 \cdot 3$ & 11.9 & $2 \cdot 3$ & 11.6 & $2 \cdot 0$ & $11 \cdot 1^{*}$ & 1.9 & $12 \cdot 0$ & $2 \cdot 4$ & 11.7 & $2 \cdot 3$ & 11.7 & $2 \cdot 1$ & $11 \cdot 4^{*}$ & 1.8 \\
\hline SFA (E\%) & 18.5 & 4.4 & $18 \cdot 1$ & 3.8 & $18 \cdot 0$ & 3.8 & $17 \cdot 9^{*}$ & 3.6 & $16 \cdot 6$ & 3.6 & 17.5 & 3.4 & $18 \cdot 3$ & 3.7 & $20 \cdot 1^{\star}$ & $4 \cdot 2$ \\
\hline Unprocessed red meat $(\mathrm{g} / \mathrm{d})$ & 75 & 52 & 79 & 50 & 70 & 46 & 78 & 55 & 79 & 52 & 75 & 51 & 71 & 50 & 78 & 50 \\
\hline Processed red meat $(\mathrm{g} / \mathrm{d})$ & 71 & 62 & 72 & 68 & 68 & 55 & 69 & 57 & 69 & 65 & 71 & 62 & 69 & 58 & 71 & 57 \\
\hline $\begin{array}{l}\text { Fruits, berries and } \\
\text { vegetables }(\mathrm{g} / \mathrm{d})\end{array}$ & 220 & 142 & 266 & 158 & 276 & 168 & $272^{*}$ & 155 & 277 & 171 & 271 & 158 & 254 & 149 & $232^{*}$ & 147 \\
\hline $\mathrm{Ca}$ intake $(\mathrm{mg} / \mathrm{d})$ & 1139 & 371 & 1213 & 341 & 1314 & 313 & $1508^{*}$ & 356 & 1088 & 340 & 1219 & 332 & 1326 & 323 & $1540^{*}$ & 341 \\
\hline
\end{tabular}

${ }^{*} P_{\text {for trend }}$ across quartiles $<0.05 ; P_{\text {for trend }}$ was assessed with linear regression (continuous variables) or with $x^{2}$ test (categorical variables). 
$67 \%(477(\mathrm{sD} 333) \mathrm{g} / \mathrm{d})$ of the total dairy product intake, $87 \%$ of the fermented dairy product intake and $60 \%$ of the nonfermented dairy product intake. Sour milk accounted for $84 \%$ of the low-fat fermented dairy product intake, cheese $88 \%$ of the high-fat fermented dairy product intake, low-fat milk $99.9 \%$ of the low-fat non-fermented dairy product intake and high-fat milk $88 \%$ of the high-fat non-fermented dairy product intake. The mean butter intake was 33 (SD 27) g/d.

Over a mean follow-up time of $20 \cdot 1$ years (minimum-maximum 0.1-28.8 years, 39882 person-years), 472 fatal or non-fatal first incident CHD events occurred. Table 3 illustrates HR for dairy product consumption, using the lowest quartile as the reference. Total dairy food intake had no association with the risk of incident CHD but fermented and non-fermented subgroups had opposite associations with the risk. After multivariable-adjustments (model 2), men in the highest quartile of fermented dairy product intake had a $27 \%(95 \%$ CI 5, 44; $P$-trend $=0.02)$ reduction in risk of incident $\mathrm{CHD}$. The $\mathrm{AR}$ reduction in the highest quartile was $7 \cdot 2 \%$ (AR in the lowest quartile $=26.5 \%$ ). In contrast, men in the highest quartile of non-fermented dairy product intake had a $52 \%$ (95\% CI 13, 104; $P$-trend $=0.003)$ increase in risk of incident $\mathrm{CHD}$. The AR increase was $11.5 \%$ (AR in the lowest quartile= $22 \cdot 0 \%$ ). Adjusting model 2 for potential effect mediators (BMI, hypertension and type 2 diabetes) had no appreciable effect (model 3). We also conducted non-fermented dairy analysis excluding participants ( $n$ 18) with extremely high intake ( $>1500 \mathrm{~g} / \mathrm{d}$ ); this had no effect on the results (data not shown). Neither did adjusting model 2 further for energy-adjusted intake of SFA, living area (rural/city), income, nor marital status (data not shown). Butter intake was not associated with risk of incident CHD, especially after multivariate adjustments (Table 3).

When evaluated continuously, each $100 \mathrm{~g}$ increase in intake of fermented dairy products was associated with a $7 \%$ lower risk (95\% CI 2, 11; model 2) and each $100 \mathrm{~g}$ increase in intake of nonfermented dairy products was associated with a $5 \%$ (95\% CI 1, 8) higher risk of incident CHD. Fig. 1 shows cumulative survival and hazard curves of $\mathrm{CHD}$ for fermented and non-fermented dairy product intake quartiles.

After dividing fermented and non-fermented dairy products further into low-fat and high-fat subgroups (Table 4), only lowfat fermented dairy product intake had a statistically significant association with the incident CHD risk, with 26\% (95\% CI 3, 43; $P$-trend $=0.03)$ decrease in risk when comparing the highest quartile to the lowest (AR in the lowest quartile $=25 \cdot 1 \%$, AR reduction in the highest quartile $=6.5 \%)$. Total low-fat $(\mathrm{HR}=$ $0 \cdot 84,95 \%$ CI 0.65, 1.09; $P$-trend $=0 \cdot 27)$ or high-fat $(\mathrm{HR}=1 \cdot 06$,

Table 3. Intake of dairy products and risk of incident $\mathrm{CHD}$

(Hazard ratios (HR) and $95 \%$ confidence intervals)

\begin{tabular}{|c|c|c|c|c|c|c|c|c|}
\hline & \multicolumn{7}{|c|}{ Intake quartile } & \multirow[b]{3}{*}{$P$-trend } \\
\hline & \multirow[b]{2}{*}{1} & \multicolumn{2}{|r|}{2} & \multicolumn{2}{|c|}{3} & \multicolumn{2}{|c|}{4} & \\
\hline & & $\mathrm{HR}$ & $95 \% \mathrm{Cl}$ & $\mathrm{HR}$ & $95 \% \mathrm{Cl}$ & $\mathrm{HR}$ & $95 \% \mathrm{Cl}$ & \\
\hline \multicolumn{9}{|l|}{ Total dairy products } \\
\hline Median intake (g/d) & 280 & \multicolumn{2}{|r|}{568} & \multicolumn{2}{|c|}{799} & \multicolumn{2}{|c|}{1115} & \\
\hline Events/participants $(n)$ & $112 / 495$ & \multicolumn{2}{|r|}{$113 / 495$} & \multicolumn{2}{|c|}{$125 / 496$} & \multicolumn{2}{|c|}{$122 / 495$} & \\
\hline Incidence rate/1000 PY & 11.2 & \multicolumn{2}{|r|}{11.1} & \multicolumn{2}{|c|}{$12 \cdot 6$} & \multicolumn{2}{|c|}{12.5} & \\
\hline Model $1^{*}$ & 1 & 0.97 & $0.74,1.26$ & 1.07 & $0.82,1.40$ & $1 \cdot 11$ & $0.82,1.48$ & 0.41 \\
\hline Model $2 \dagger$ & 1 & 0.94 & $0.72,1.22$ & 1.01 & $0.77,1.33$ & 1.03 & $0.75,1.42$ & 0.75 \\
\hline Model 3¥ & 1 & 0.93 & $0.71,1.21$ & 0.96 & $0.73,1.27$ & 0.97 & $0.71,1.34$ & 0.92 \\
\hline \multicolumn{9}{|l|}{ Fermented dairy products } \\
\hline Median intake $(\mathrm{g} / \mathrm{d})$ & 5 & \multicolumn{2}{|r|}{58} & \multicolumn{2}{|c|}{177} & \multicolumn{2}{|c|}{436} & \\
\hline Events/participants $(n)$ & $133 / 501$ & \multicolumn{2}{|r|}{$117 / 494$} & \multirow{2}{*}{\multicolumn{2}{|c|}{$\begin{array}{c}121 / 492 \\
11.9\end{array}$}} & \multicolumn{2}{|c|}{$101 / 494$} & \\
\hline Incidence rate/1000 PY & $14 \cdot 1$ & \multicolumn{2}{|r|}{11.4} & & & \multicolumn{2}{|c|}{$10 \cdot 0$} & \\
\hline Model $1^{\star}$ & 1 & 0.84 & $0.65,1.07$ & 0.84 & $0.65,1.07$ & 0.67 & $0.52,0.87$ & 0.01 \\
\hline Model $2 \dagger$ & 1 & 0.93 & $0.72,1.20$ & 0.93 & $0.73,1 \cdot 20$ & 0.73 & $0.56,0.95$ & 0.02 \\
\hline Model $3 \ddagger$ & 1 & 1.03 & $0.80,1.32$ & 0.96 & $0.75,1.24$ & 0.74 & $0.57,0.97$ & 0.01 \\
\hline \multicolumn{9}{|c|}{ Non-fermented dairy products } \\
\hline Median intake $(\mathrm{g} / \mathrm{d})$ & 156 & \multicolumn{2}{|r|}{366} & \multicolumn{2}{|c|}{581} & & & \\
\hline Events/participants $(n)$ & $109 / 495$ & & $105 / 495$ & & 496 & & 495 & \\
\hline Incidence rate/1000 PY & $10 \cdot 7$ & & $10 \cdot 3$ & & & & & \\
\hline Model $1^{*}$ & 1 & 0.97 & $0.74,1.27$ & 1.05 & $0.80,1.38$ & 1.59 & $1 \cdot 21,2 \cdot 10$ & $<0.001$ \\
\hline Model $2 \dagger$ & 1 & 0.95 & $0.72,1.25$ & 1.02 & $0.78,1.34$ & 1.52 & $1.13,2.04$ & 0.003 \\
\hline Model 3ł & 1 & 0.92 & $0 \cdot 70,1 \cdot 21$ & 0.96 & $0.73,1.26$ & 1.43 & $1.07,1.92$ & 0.01 \\
\hline Butter & & & & & & & & \\
\hline Median intake (g/d) & 4 & & 20 & & & & 3 & \\
\hline Events/participants $(n)$ & $103 / 495$ & & $117 / 495$ & & 496 & & 495 & \\
\hline Incidence rate/1000 PY & $10 \cdot 2$ & & 11.5 & & & & & \\
\hline Model $1^{\star}$ & 1 & $1 \cdot 10$ & $0.84,1.44$ & 1.12 & $0.85,1.47$ & 1.31 & $0.97,1.76$ & 0.08 \\
\hline Model $2 \dagger$ & 1 & 1.10 & $0.83,1.46$ & 1.04 & $0.77,1.40$ & 1.15 & $0.80,1.64$ & 0.55 \\
\hline Model 3‡ & 1 & 1.11 & $0.84,1.47$ & 1.09 & $0.81,1.47$ & 1.14 & $0.80,1.64$ & 0.55 \\
\hline
\end{tabular}

PY, person-years.

* Model 1 adjusted for age, examination year and energy intake.

† Model 2 adjusted for model 1 and pack-years of smoking, leisure-time physical activity, years of education, family history of ischaemic heart disease, intakes of alcohol, fruits, berries and vegetables, fibre and percentage of energy from PUFA.

‡ Model 3 adjusted for model 2 and BMI, diabetes and hypertension. 

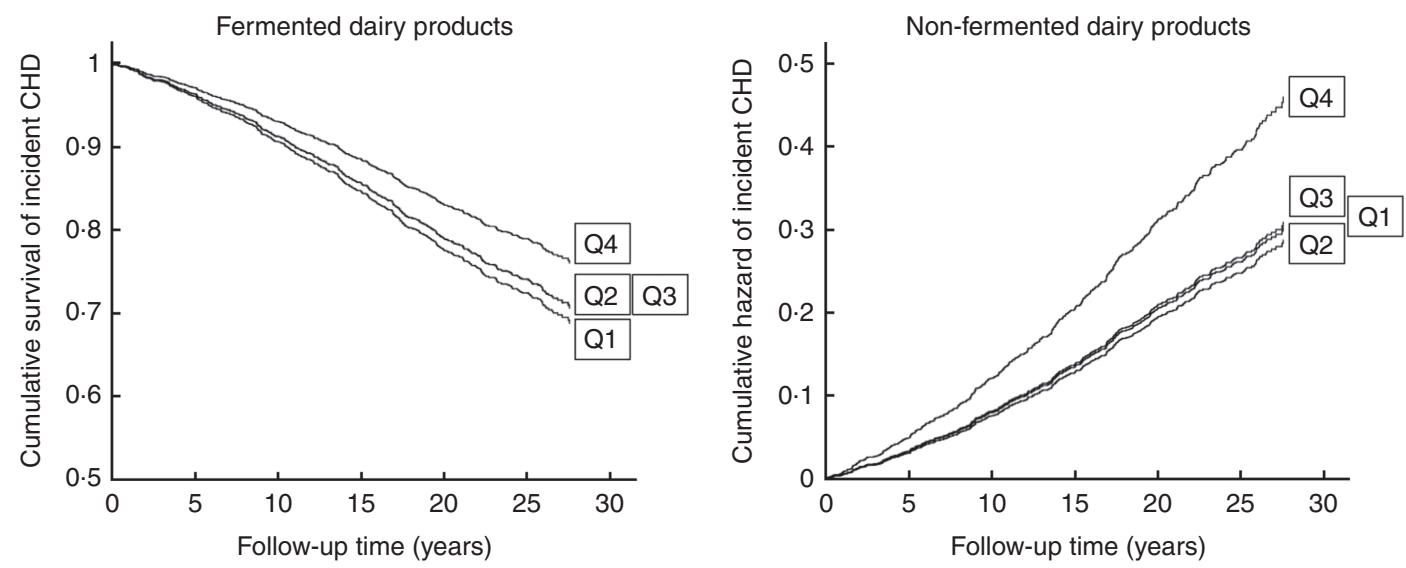

Fig. 1. Cumulative survival or hazard of incident CHD according to quartiles (Q) of fermented and non-fermented dairy product intake in 1981 middle-aged and older men from the Kuopio Ischaemic Heart Disease Risk Factor Study.

$95 \%$ CI $0 \cdot 80,1 \cdot 42 ; P$-trend $=0 \cdot 37$ ) dairy product intakes had no association with the risk.

In the more detailed subgroup analyses, we investigated whether milk or cheese intakes were associated with the risk of incident CHD. Milk comprised 95\% (494 (SD 331) g/d) of the total non-fermented dairy product intake. Total milk intake was associated with a higher risk of incident CHD. Men in the highest intake quartile $(>700 \mathrm{~g} / \mathrm{d})$ had $48 \%$ (95\% CI 10, 97; $P$-trend $=$ 0.004; model 2) higher risk, when compared with the lowest quartile $(<235 \mathrm{~g} / \mathrm{d}$; AR in the lowest quartile $=22.2 \%$, AR increase in the highest quartile $=10 \cdot 7 \%$ ). When assessed separately, neither low-fat nor high-fat milk intake was significantly associated with the risk after multivariable adjustments (Table 4). Cheese intake (22 (SD 26) g/d) had an inverse association with $\mathrm{CHD}$ risk in the basic model, but this association was attenuated after multivariate adjustments (Table 4).

In the substitution models, replacing $100 \mathrm{~g}$ of non-fermented dairy product with an equal amount of fermented dairy product was associated with $8 \%$ lower risk of incident CHD (HR 0.92, 95\% CI 0.87, 0.96; model 2). Substituting low-fat non-fermented with low-fat fermented dairy product yielded similar results (HR $0 \cdot 93,95 \%$ CI $0 \cdot 89,0 \cdot 99)$.

Investigating associations between the dairy product intakes and risk of CHD incidence with a shorter, 11-year follow-up time (199 events) provided results comparable to our main analyses. Comparing highest intake quartile to the lowest, HR were 0.97 (95\% CI $0.61,1.56 ; P$-trend $=0.97 ;$ model 2$)$ for total dairy products, 0.76 (95\% CI $0.51,1.13 ; P$-trend $=0.25)$ for fermented dairy products, and 1.76 (95\% CI 1.11, 2.80; $P$-trend $=0.02)$ for non-fermented dairy products. The HR for butter was $0.99(95 \%$ CI $0.57,1.72 ; P$-trend $=0.85)$ (other data not shown).

\section{Discussion}

In this prospective, population-based cohort study, we examined whether total dairy product intake or its several subgroups were associated with the risk of incident CHD in middle-aged and older men from Eastern Finland, a population with a high dairy product intake. Total dairy product intake had no association with the CHD risk, but in the subgroup analyses, fermented dairy products had an inverse association whereas non-fermented dairy products had a direct association with the risk. Further analyses of low-fat and high-fat categories showed that only lowfat fermented dairy product intake was inversely associated with the $\mathrm{CHD}$ risk. Butter or cheese were not associated with the risk, but total milk intake was associated with a higher risk. Neither lowfat nor high-fat milk consumption was associated with the CHD risk, however.

Our results suggest that fermentation affects the association between dairy product consumption and risk of CHD. Previous studies provide little evidence regarding the association between total fermented dairy product intake and risk of CHD. Cohort studies from Netherlands ${ }^{(27,28)}$ and England ${ }^{(29)}$ showed a similar inverse trend to our findings, but with non-significant associations. The median fermented dairy product intakes in those studies ranged from 41 to $147 \mathrm{~g} / \mathrm{d}^{(27-29)}$, whereas in our study the median intake was $105 \mathrm{~g} / \mathrm{d}$ with a large variation (interquartile ranges $25-280 \mathrm{~g} / \mathrm{d}$ ). In addition, the results may differ due to variation in products consumed as fermented dairy foods. For example, in the KIHD, low-fat fermented dairy products comprised mostly of sour milk and, in much lower amounts, of low-fat yogurt and cultured buttermilk known as viili $^{(30)}$, and high-fat fermented dairy products were mainly cheese, whereas yogurt comprised a significant amount of the fermented dairy product intake in the Dutch cohorts ${ }^{(27,31)}$. As two previous meta-analyses found no significant association between yogurt consumption and CHD risk ${ }^{(21,32)}$, the type of fermented dairy food might also matter. Even though yogurt, viili and sour milk are somewhat similar products regarding fermentation, fat content and viscosity, the different ways these products are consumed might affect the outcomes. In Finland, for example, sour milk has been a common lunch or dinner beverage, whereas yogurt and viili are snack-like products and can be often combined with berries, muesli or added sugar, for example. These combinations are difficult to account for in statistical analyses but could have divergent effects on health. Based on epidemiological evidence ${ }^{(33)}$, including our results, fermented dairy foods could be beneficial for cardiovascular health, but more studies are needed to confirm this.

The existing evidence for non-fermented dairy products mainly concentrates on milk, which is also the main source of 
Table 4. Intake of low-fat and high-fat dairy products and risk of incident CHD (Hazard ratios (HR) and $95 \%$ confidence intervals)

\begin{tabular}{|c|c|c|c|c|c|c|c|c|}
\hline & \multicolumn{7}{|c|}{ Intake quartile } & \multirow[b]{3}{*}{$P$-trend } \\
\hline & \multirow[b]{2}{*}{1} & \multicolumn{2}{|r|}{2} & \multicolumn{2}{|r|}{3} & \multicolumn{2}{|c|}{4} & \\
\hline & & HR & $95 \% \mathrm{Cl}$ & HR & $95 \% \mathrm{Cl}$ & HR & $95 \% \mathrm{Cl}$ & \\
\hline \multicolumn{9}{|l|}{ Fermented dairy products } \\
\hline \multicolumn{9}{|c|}{ Low-fat fermented dairy products } \\
\hline Median intake $(\mathrm{g} / \mathrm{d})$ & 0 & & 50 & & 189 & & 53 & \\
\hline Events/participants $(n)$ & $181 / 722$ & & $102 / 422$ & & $104 / 419$ & & 418 & \\
\hline Incidence rate/1000 PY & $12 \cdot 8$ & & 11.8 & & 11.9 & & $\cdot 1$ & \\
\hline Model $1^{*}$ & 1 & 0.90 & $0.70,1.14$ & 0.86 & $0.68,1.10$ & 0.72 & $0.55,0.93$ & 0.02 \\
\hline Model $2 \dagger$ & 1 & 0.96 & $0.75,1.23$ & 0.94 & $0.73,1.19$ & 0.74 & $0.57,0.97$ & 0.03 \\
\hline Model $3 \ddagger$ & 1 & 1.00 & $0.78,1.27$ & 0.94 & $0.74,1.20$ & 0.72 & $0.56,0.94$ & 0.01 \\
\hline \multicolumn{9}{|c|}{ High-fat fermented dairy products } \\
\hline Median intake $(\mathrm{g} / \mathrm{d})$ & 0 & & 8 & & 25 & & 5 & \\
\hline Events/participants $(n)$ & $119 / 466$ & & $139 / 516$ & & $118 / 500$ & & 499 & \\
\hline Incidence rate/1000 PY & $13 \cdot 8$ & & 13.6 & & $11 \cdot 2$ & & $\cdot 2$ & \\
\hline Model $1^{*}$ & 1 & 1.00 & $0.78,1.27$ & 0.85 & $0.66,1.10$ & 0.75 & $0.57,0.99$ & 0.02 \\
\hline Model 2† & 1 & $1 \cdot 10$ & $0.86,1.41$ & 1.00 & $0.77,1.30$ & 0.93 & $0 \cdot 70,1 \cdot 23$ & 0.36 \\
\hline Model 3‡ & 1 & $1 \cdot 16$ & $0.91,1.49$ & 1.05 & $0.81,1.37$ & 1.01 & $0.76,1.35$ & $0 \cdot 71$ \\
\hline \multicolumn{9}{|l|}{ Cheese } \\
\hline Median intake $(\mathrm{g} / \mathrm{d})$ & 0 & & 9 & & 24 & & 0 & \\
\hline Events/participants $(n)$ & $135 / 515$ & & $135 / 529$ & & $107 / 456$ & & 481 & \\
\hline Incidence rate/1000 PY & $14 \cdot 1$ & & $12 \cdot 8$ & & $11 \cdot 1$ & & 4 & \\
\hline Model $1^{*}$ & 1 & 0.91 & $0.72,1.15$ & 0.83 & $0.64,1.07$ & 0.77 & $0.59,1.00$ & 0.05 \\
\hline Model 2† & 1 & 1.02 & $0.80,1.30$ & 0.98 & $0.75,1.27$ & 0.96 & $0.72,1.27$ & 0.70 \\
\hline Model 3‡ & 1 & 1.07 & $0.84,1.37$ & 1.04 & $0.80,1.35$ & 1.03 & $0.78,1.36$ & 0.96 \\
\hline \multicolumn{9}{|c|}{ Non-fermented dairy products } \\
\hline \multicolumn{9}{|c|}{ Low-fat non-fermented dairy products } \\
\hline Median intake $(\mathrm{g} / \mathrm{d})$ & 37 & & 140 & & 346 & & 78 & \\
\hline Events/participants $(n)$ & $119 / 495$ & & $118 / 495$ & & $116 / 496$ & & /495 & \\
\hline Incidence rate/1000 PY & $12 \cdot 3$ & & 11.8 & & 11.4 & & 1.9 & \\
\hline Model $1^{*}$ & 1 & 0.98 & $0.76,1.27$ & 0.98 & $0.76,1.26$ & 1.03 & $0.80,1.33$ & 0.76 \\
\hline Model $2 \dagger$ & 1 & $1 \cdot 11$ & $0.85,1.44$ & 1.08 & $0.83,1.41$ & $1 \cdot 17$ & $0.90,1.52$ & $0 \cdot 31$ \\
\hline Model 3‡ & 1 & 1.09 & $0.84,1.41$ & 1.02 & $0.79,1.33$ & $1 \cdot 12$ & $0.86,1.46$ & 0.51 \\
\hline \multicolumn{9}{|l|}{ Low-fat milk } \\
\hline Median intake $(\mathrm{g} / \mathrm{d})$ & 37 & & 140 & & 346 & & 78 & \\
\hline Events/participants $(n)$ & $118 / 495$ & & $118 / 495$ & & $117 / 496$ & & /495 & \\
\hline Incidence rate/1000 PY & $12 \cdot 2$ & & $11 \cdot 8$ & & 11.5 & & 1.9 & \\
\hline Model $1^{*}$ & 1 & 0.99 & $0.77,1.28$ & 1.00 & $0.77,1.29$ & 1.04 & $0.81,1.35$ & 0.71 \\
\hline Model $2 \dagger$ & 1 & $1 \cdot 12$ & $0.86,1.45$ & $1 \cdot 10$ & $0.85,1.43$ & $1 \cdot 18$ & $0.91,1.54$ & 0.28 \\
\hline Model 3‡ & 1 & $1 \cdot 10$ & $0.85,1.43$ & 1.04 & $0.80,1.35$ & $1 \cdot 13$ & $0.87,1.47$ & 0.47 \\
\hline \multicolumn{9}{|c|}{ High-fat non-fermented dairy products } \\
\hline Median intake $(\mathrm{g} / \mathrm{d})$ & 2 & & 31 & & 129 & & 83 & \\
\hline Events/participants $(n)$ & $108 / 495$ & & $103 / 495$ & & $123 / 496$ & & /495 & \\
\hline Incidence rate/1000 PY & 11.0 & & $9 \cdot 8$ & & $12 \cdot 1$ & & 4.6 & \\
\hline Model $1^{*}$ & 1 & 0.87 & $0.67,1.14$ & 1.04 & $0.80,1.36$ & $1 \cdot 25$ & $0.95,1.65$ & 0.02 \\
\hline Model $2 \dagger$ & 1 & 0.91 & $0.70,1.20$ & 1.05 & $0.81,1.37$ & $1 \cdot 12$ & $0.83,1.50$ & 0.29 \\
\hline Model 3‡ & 1 & 0.95 & $0.72,1.25$ & $1 \cdot 10$ & $0.84,1.43$ & $1 \cdot 14$ & $0.85,1.54$ & 0.30 \\
\hline \multicolumn{9}{|l|}{ High-fat milk } \\
\hline Median intake (g/d) & 0 & & 21 & & 185 & & 45 & \\
\hline Events/participants $(n)$ & $181 / 818$ & & $80 / 388$ & & $98 / 388$ & & /387 & \\
\hline Incidence rate/1000 PY & $10 \cdot 7$ & & $10 \cdot 0$ & & $12 \cdot 7$ & & 5.5 & \\
\hline Model $1^{*}$ & 1 & 0.90 & $0.69,1.17$ & 1.09 & $0.85,1.41$ & $1 \cdot 38$ & $1.07,1.78$ & 0.004 \\
\hline Model $2 \dagger$ & 1 & 0.91 & $0.70,1 \cdot 19$ & 1.02 & $0.79,1.33$ & $1 \cdot 19$ & $0.90,1.57$ & 0.14 \\
\hline Model 3‡ & 1 & 0.94 & $0.72,1.23$ & 1.06 & $0.81,1.37$ & $1 \cdot 19$ & $0.90,1.57$ & 0.16 \\
\hline
\end{tabular}

PY, person-years.

* Model 1 adjusted for age, examination year and energy intake.

† Model 2 adjusted for model 1 and pack-years of smoking, leisure-time physical activity, years of education, family history of ischaemic heart disease, intakes of alcohol, fruits, berries and vegetables, fibre and percentage of energy from PUFA.

¥ Model 3 adjusted for model 2 and BMl, diabetes and hypertension.

non-fermented dairy products in the KIHD. Meta-analyses studying total milk consumption show neutral ${ }^{(21,33-35)}$ or inverse $^{(36)}$ association with the risk of CHD. Similar associations have been reported for CVD, but the evidence is more inconsistent ${ }^{(9)}$. These results do not support our finding of increased CHD risk with non-fermented dairy product or total milk intake. This controversy might be caused by differences in non-fermented dairy product consumption. Median intakes in most previous cohort studies have ranged between 62 and $442 \mathrm{~g} / \mathrm{d}^{(37)}$, whereas in the KIHD, the median for 
non-fermented dairy product consumption was $465 \mathrm{~g} / \mathrm{d}$ for the whole study population and $907 \mathrm{~g} / \mathrm{d}$ for the highest intake quartile. A total of three studies from Sweden with comparable non-fermented dairy product or milk intakes also report positive associations on CVD mortality ${ }^{(38)}$ and all-cause mortality ${ }^{(38,39)}$, and a trend towards higher risk of myocardial infarction $^{(40)}$. In our analyses, low-fat and high-fat non-fermented dairy products did not have statistically significant associations with the CHD risk. This suggests that only a very high non-fermented dairy product intake increases the risk of $\mathrm{CHD}$. Whether this is due to the intake of non-fermented dairy products themselves or due to other factors associated with a very high non-fermented dairy product intake, remains to be investigated. In our cohort, the participants in the highest intake quartile of non-fermented dairy products also had unhealthier lifestyle, including unhealthier diet, which could at least partly explain the findings. High SFA intake could also explain the observed association with total non-fermented dairy product intake but adjusting multivariate models further for SFA intake had no effect on the association. These results should be interpreted with care, because the KIHD represents a unique population, where milk is widely used with different food items and it is a common beverage among these men. In addition, the average milk consumption in Finland has almost halved after 1975, being about $122 \mathrm{~kg} /$ person per year in $2016^{(3)}$. Therefore, we cannot confirm how well the milk consumption patterns from baseline represent the whole follow-up period

Even though fermented and non-fermented dairy products had opposite associations with the risk of $\mathrm{CHD}$, cheese and butter, products rich in dairy fat, were not associated with the risk. These findings are comparable to previous meta-analyses, which did not find an increased CHD risk with higher cheese or butter intake $^{(10,11)}$. Although butter consumption in the KIHD is higher than in most cohorts ${ }^{(11)}$, we observed a neutral association with the CHD risk. In the basic model butter had a trend towards a higher risk and cheese towards a lower risk, but both associations were significantly attenuated after adjustments for potential confounders. This suggest that other lifestyle and dietary factors associated with a higher intake of these products explain the majority of the associations in the minimally adjusted models. We have previously reported a statistically significant, multivariable-adjusted, $65 \%$ risk increase of incident CHD in the highest butter intake quartile in this same study population, but with a 7 years shorter follow-up ${ }^{(41)}$. In the current analyses, however, we did not find an association with a shorter, 10-year follow-up, either. This suggests that the lack of an association in the main analysis is not due to the long follow-up. Such a sensitivity to the length of follow-up is an interesting observation, but overall our findings suggest that butter intake is not independently associated with the risk of CHD even in this population with high butter consumption.

The potentially harmful effects of SFA in some dairy products might be blunted by the so-called 'dairy matrix'. This matrix involves all nutrients found in dairy products, such as $\mathrm{Ca}$, and $\mathrm{P}$, and factors related to processing methods, such as different starter cultures of fermented dairy products and effects on milk fat globule membranes. The effect of the matrix has been observed with cheese intake, which in clinical trials has produced an attenuated effect on blood total and LDL-cholesterol when compared with butter, despite similar amounts of SFA ${ }^{(15)}$. A few proposed reasons for this difference are decreased fatty acid absorption, increased bile acid excretion, or changes in SCFA metabolism caused by the matrix ${ }^{(16,42)}$. Our results support this theory, as cheese intake was not associated with increased CHD risk. However, because only low-fat fermented dairy product intake had a beneficial association with CHD risk and high consumption of non-fermented dairy products was associated with an increased risk of $\mathrm{CHD}$, we cannot completely rule out the possibly harmful effect of dairy SFA.

Main strengths of this study include the population-based recruitment, extensive database of potential confounders, detailed classification of the CHD events, and virtually no losses to follow-up. The high dairy product consumption among the study population and detailed information on different dairy products consumed enabled us to separate fermented and non-fermented dairy product intake and to form multiple subcategories. However, measuring the diet only with one 4-d diet record collected at baseline could cause misclassification and random error, which would attenuate the associations between the dietary factors and risk of incident events with a long followup. Therefore, we also examined the associations with the shorter 11-year follow-up, which provided generally similar results. Dividing fermented and non-fermented dairy products into low-fat and high-fat groups resulted in high-fat fermented dairy analyses to be mainly driven by cheese and low-fat nonfermented dairy analyses by milk. As such, we were not able to investigate how other high-fat fermented or low-fat nonfermented dairy products are associated with CHD. Also, because only few participants consumed fat-free dairy products at baseline, we could not use them as a separate category in our analyses. Finally, our study population included only Caucasian men, restricting the generalisability of the study findings to women or to other races and ethnicities. And, even though we adjusted the multivariate models for several confounding factors, we cannot rule out possible residual confounding.

To conclude, we found that high intakes of fermented and especially low-fat fermented dairy products had an inverse association with the risk of $\mathrm{CHD}$, whereas very high intakes of non-fermented dairy products and milk were associated with increased risk of CHD. These findings suggest that fermentation could be one key element affecting the associations between different dairy products and the development of CHD.

\section{Acknowledgements}

T. T. K. has received funding for this study from the following foundations: Finnish Foundation for Cardiovascular Research; Otto A. Malm Foundation; Olvi Foundation; Yrjö Jahnsson Foundation (grant no. 6852); and North Savo Regional Fund. The KIHD project was funded by a large number of research grants to Professor Jukka T. Salonen and his co-workers. These funding agencies had no role in the study design, analysis, decision to publish or writing of this article.

The authors' responsibilities are as follows: T. T. K. analysed the data and drafted the manuscript; J. K. V. analysed the data 
and had primary responsibility for final content; T. T. K., H. E. K. V., S. V., T.-P. T., J. M. and J. K. V. acquired data, designed and conducted research; H. E. K. V., S. V., T.-P. T., J. M. and J. K. V. critically revised the manuscript for important intellectual content. All authors read and approved the final manuscript.

The authors declare that there are no conflicts of interest.

\section{Supplementary material}

For supplementary material/s referred to in this article, please visit https://doi.org/10.1017/S0007114518002830

\section{References}

1. World Health Organization (2017) Cardiovascular diseases. http://www.who.int/mediacentre/factsheets/fs317/en/ (accessed September 2017).

2. Puska P, Vartiainen E, Nissinen A, et al. (2016) Background, principles, implementation, and general experiences of the North Karelia Project. Glob Heart 11, 173-178.

3. Natural Resources Institute Finland (2017) Balance sheet for food commodities. http://stat.luke.fi/en/balance $\% 20$ sheet $\%$ 20for\%20food\%20commodities (accessed September 2017).

4. Puska P (2009) Fat and heart disease: yes we can make a change - the case of North Karelia (Finland). Ann Nutr Metab 54, 33-38.

5. Schwab U, Lauritzen L, Tholstrup T, et al. (2014) Effect of the amount and type of dietary fat on cardiometabolic risk factors and risk of developing type 2 diabetes, cardiovascular diseases, and cancer: a systematic review. Food Nutr Res 58, 10.3402/fnr.v58.25145.

6. de Oliveira Otto MC, Mozaffarian D, Kromhout D, et al. (2012) Dietary intake of saturated fat by food source and incident cardiovascular disease: the Multi-Ethnic Study of Atherosclerosis. Am J Clin Nutr 96, 397-404.

7. Chen M, Li Y, Sun Q, et al. (2016) Dairy fat and risk of cardiovascular disease in 3 cohorts of US adults. Am J Clin Nutr 104, 1209-1217.

8. de Oliveira Otto MC, Lemaitre RN, Song X, et al. (2018) Serial measures of circulating biomarkers of dairy fat and total and cause-specific mortality in older adults: the Cardiovascular Health Study. Am J Clin Nutr 108, 476-484.

9. Drouin-Chartier JP, Brassard D, Tessier-Grenier $\mathrm{M}$, et al. (2016) Systematic review of the association between dairy product consumption and risk of cardiovascular-related clinical outcomes. Adv Nutr 7, 1026-1040.

10. Chen GC, Wang Y, Tong X, et al. (2017) Cheese consumption and risk of cardiovascular disease: a meta-analysis of prospective studies. Eur J Nutr 56, 2565-2575.

11. Pimpin L, Wu JH, Haskelberg H, et al. (2016) Is butter back? A systematic review and meta-analysis of butter consumption and risk of cardiovascular disease, diabetes, and total mortality. PLOS ONE 11, e0158118.

12. Steinmetz KA, Childs MT, Stimson C, et al. (1994) Effect of consumption of whole milk and skim milk on blood lipid profiles in healthy men. Am J Clin Nutr 59, 612-618.

13. Engel S, Elhauge M \& Tholstrup T (2018) Effect of whole milk compared with skimmed milk on fasting blood lipids in healthy adults: a 3-week randomized crossover study. Eur J Clin Nutr 72, 249-254.

14. Raziani F, Tholstrup T, Kristensen MD, et al. (2016) High intake of regular-fat cheese compared with reduced-fat cheese does not affect LDL cholesterol or risk markers of the metabolic syndrome: a randomized controlled trial. Am J Clin Nutr 104, 973-981.

15. de Goede J, Geleijnse JM, Ding EL, et al. (2015) Effect of cheese consumption on blood lipids: a systematic review and meta-analysis of randomized controlled trials. Nutr Rev 73, 259-275.

16. Thorning TK, Bertram HC, Bonjour JP, et al. (2017) Whole dairy matrix or single nutrients in assessment of health effects: current evidence and knowledge gaps. Am J Clin Nutr $\mathbf{1 0 5}$, 1033-1045.

17. Tapsell LC (2015) Fermented dairy food and CVD risk. Br J Nutr 113, Suppl. 2, 131-135.

18. Mozaffarian D \& Wu JHY (2018) Flavonoids, dairy foods, and cardiovascular and metabolic health: a review of emerging biologic pathways. Circ Res 122, 369-384.

19. Gast GC, de Roos NM, Sluijs I, et al. (2009) A high menaquinone intake reduces the incidence of coronary heart disease. Nutr Metab Cardiovasc Dis 19, 504-510.

20. Geleijnse JM, Vermeer C, Grobbee DE, et al. (2004) Dietary intake of menaquinone is associated with a reduced risk of coronary heart disease: the Rotterdam Study. J Nutr 134, 3100-3105.

21. Alexander DD, Bylsma LC, Vargas AJ, et al. (2016) Dairy consumption and CVD: a systematic review and metaanalysis. Br J Nutr 115, 737-750.

22. Singh GM, Micha R, Khatibzadeh S, et al. (2015) Global, regional, and national consumption of sugar-sweetened beverages, fruit juices, and milk: a systematic assessment of beverage intake in 187 countries. PLOS ONE 10, e0124845.

23. Salonen JT (1988) Is there a continuing need for longitudinal epidemiologic research? The Kuopio Ischaemic Heart Disease Risk Factor Study. Ann Clin Res 20, 46-50.

24. Salonen JT, Nyyssonen K, Korpela H, et al. (1992) High stored iron levels are associated with excess risk of myocardial infarction in eastern Finnish men. Circulation 86, 803-811.

25. Lakka TA, Venalainen JM, Rauramaa R, et al. (1994) Relation of leisure-time physical activity and cardiorespiratory fitness to the risk of acute myocardial infarction in men. $N$ Engl J Med 330, 1549-1554.

26. Laaksonen DE, Lakka TA, Lakka HM, et al. (2002) Serum fatty acid composition predicts development of impaired fasting glycaemia and diabetes in middle-aged men. Diabet Med 19, 456-464.

27. Praagman J, Franco OH, Ikram MA, et al. (2015) Dairy products and the risk of stroke and coronary heart disease: the Rotterdam Study. Eur J Nutr 54, 981-990.

28. Dalmeijer GW (2013) Dairy intake and coronary heart disease or stroke-a population-based cohort study. Int J Cardiol 167, 925-929.

29. Soedamah-Muthu SS, Masset G, Verberne L, et al. (2013) Consumption of dairy products and associations with incident diabetes, CHD and mortality in the Whitehall II study. $\mathrm{Br} J$ Nutr 109, 718-726.

30. Kahala M, Maki M, Lehtovaara A, et al. (2008) Characterization of starter lactic acid bacteria from the Finnish fermented milk product viili. J Appl Microbiol 105, 1929-1938.

31. Praagman J, Dalmeijer GW, van der Schouw YT, et al. (2015) The relationship between fermented food intake and mortality risk in the European Prospective Investigation into Cancer and Nutrition-Netherlands cohort. Br J Nutr 113, 498-506.

32. Qin LQ, Xu JY, Han SF, et al. (2015) Dairy consumption and risk of cardiovascular disease: an updated meta-analysis of prospective cohort studies. Asia Pac J Clin Nutr 24, 90-100.

33. Guo J, Astrup A, Lovegrove JA, et al. (2017) Milk and dairy consumption and risk of cardiovascular diseases and all-cause 
mortality: dose-response meta-analysis of prospective cohort studies. Eur J Epidemiol 32, 269-287.

34. Elwood PC (2004) Milk drinking, ischaemic heart disease and ischaemic stroke II. Evidence from cohort studies. Eur J Clin Nutr 58, 718-724.

35. Mullie P, Pizot C \& Autier P (2016) Daily milk consumption and all-cause mortality, coronary heart disease and stroke: a systematic review and meta-analysis of observational cohort studies. BMC Public Health 16, 1236.

36. Elwood PC, Pickering JE, Givens DI, et al. (2010) The consumption of milk and dairy foods and the incidence of vascular disease and diabetes: an overview of the evidence. Lipids 45, 925-939.

37. Gijsbers L, Ding EL, Malik VS, et al. (2016) Consumption of dairy foods and diabetes incidence: a dose-response meta-analysis of observational studies. Am J Clin Nutr 103, 1111-1124.
38. Michaelsson K, Wolk A, Langenskiold S, et al. (2014) Milk intake and risk of mortality and fractures in women and men: cohort studies. BMJ 349, g6015.

39. Tognon G, Nilsson LM, Shungin D, et al. (2017) Nonfermented milk and other dairy products: associations with all-cause mortality. Am J Clin Nutr 105, 1502-1511.

40. Patterson E, Larsson SC, Wolk A, et al. (2013) Association between dairy food consumption and risk of myocardial infarction in women differs by type of dairy food. J Nutr $\mathbf{1 4 3}$, 74-79.

41. Voutilainen S, Nurmi A, Virtanen J, et al. (2013) The use of butter and the risk of myocardial infarction. Finn Med J $\mathbf{6 8}$, 2351-2357.

42. St-Onge M (2000) Consumption of fermented and nonfermented dairy products: effects on cholesterol concentrations and metabolism. Am J Clin Nutr 71, 674-681. 